Die Pädiater können zur Zeit Fertigkeitsausweise für Hüftsonographie und Sonographie des Abdomens erwerben. Wird diese Ausbildung immer noch anerkannt bei der qualitativen Dignität, gemäss der nach TarMed abgerechnet werden kann?

Was bedeutet der Fachausweis Entwicklungspädiatrie? Genügt die Tatsache, über einen FMH-Titel Pädiatrie zu verfügen, um eine entwicklungspädiatrische Untersuchung ausserhalb der Routineuntersuchungen der SGP abzurechnen? Jeder Pädiater scheint in der Lage zu sein, eine vertiefte neuromotorische Untersuchung durchzuführen; weshalb sollen dann nur Neuropädiater dafür entschädigt werden? Im übrigen bin ich erstaunt über die wenigen pädiatrischen Positionen in TarMed; wie ist dies zu erklären?

M.-A. P. in V.

Weder Einkommen noch Bedeutung einer Fachdisziplin korrelieren mit der Anzahl von Positionen in der TarMed-Tarifstruktur. Eine Position «Pädiatrische Leistung pro Stunde à Franken 1000.-» würde zweifelsohne alle Pädiater wunschlos glücklich machen. Doch zurück zu den Realitäten:
1. Der FA Sonographie der Neugeborenenhüfte, von Gesetzes wegen vorgeschrieben, ist auch für die TarMed-Tarife uneingeschränkt gültig.

2. Der FA Entwicklungspädiatrie muss noch geschaffen werden; bis zu diesem Zeitpunkt können alle Pädiater, inkl. Besitzstandsgarantie, die Leistung verrechnen.

Zum Abschluss noch Folgendes: Die von Ihnen in Frage gestellten bzw. kritisierten Positionen wurden alle mit den Delegierten Ihrer Fachgesellschaft erarbeitet.

Wie sind die verschiedenen Röntgenräume Ultraschall gross/klein definiert?

P. W. in $K$.

Es handelt sich um Sparten, die nicht mit Räumen identisch sein müssen. Sie enthalten im wesentlichen die Geräteinvestitionskosten und Personalkosten neben Verbrauchsmaterial etc. Details können beim Tarifdienst eingesehenen werden.

\section{Questions les plus fréquentes sur le TarMed}

Médecin de premier recours à Villeneuve, je ne m'étonne pas du battage autour de TarMed. Il en va du beef-steak de chacun de nous. On nous assure que notre revenu ne devrait pas baisser sous le nouveau régime TarMed.

Ma question est des plus élémentaires: Comment peut-on le dire de bonne foi si la valeur du point n'est pas encore définie?

B. I. $\dot{a} V$.

L'affirmation en question ne vaut que pour le cas où l'introduction des tarifs serait vraiment neutre en termes de coûts. Une évaluation définitive ne sera en effet possible qu'une fois la valeur du point fixée. C'est d'ailleurs pourquoi nous pensons qu'une votation générale n'aurait aucun sens pour l'instant.

En tant que psychiatre, je dois établir le status neurologique (nerfs cérébraux, sensibilité périphérique, etc.) non seulement dans le cadre d'expertises mais aussi lorsqu'il s'agit de poser un diagnostic. Un spécialiste "étranger à la discipline" peut-il facturer cette prestation dans le TarMed?

B. M. $\dot{a} L$.
Sauf s'il peut bénéficier de la garantie des droits acquis, un spécialiste dans votre cas ne peut pas facturer cette prestation. Les prestations en question sont indemnisées via les positions de la consultation.

Une situation qui s'est présentée récemment à la clinique (dotée d'un service des urgences et collaborant avec des médecins agréés) a donné lieu à un problème d'interprétation en rapport avec le TarMed. Permettez-moi par conséquent de vous décrire la situation en question et de vous demander laquelle des solutions a) $\dot{a} d)$ ci-après serait applicable pour la facturation.

Le cas: "Vers 23 heures, un patient arrive aux urgences. Il se plaint de maux de ventre. Après un examen et des soins primaires ( $p$. ex. traitement du choc, thérapie de la douleur) par l'anesthésiste de service (médecin agréé), le problème abdominal n'étant pas clairement identifié, on appelle le spécialiste (médecin agréé). Celui-ci vient d'urgence à l'hôpital."

Question: que peut-on porter sur la facture pour la prestation médicale des deux médecins?

a) 2 consultations de base (00.0000) et d'autres consultations (0.0010) + le forfait urgence-dérangement (00.1800)?

b) 1 consultation de base / d'autres consultations et 1 consilium (36.0090) + le forfait urgence-dérangement (00.1800)?

c) 1 consultation de base et d'autres consultations + le forfait urgence-dérangement (00.1800)?

d) 1 consilium + le forfait urgence-dérangement (00.1800)?

J. G. $\dot{a} L$. 
Tout d'abord, il faudrait savoir si le médecin dont vous parlez est autorisé à facturer; ce n'est le cas que s'il est établi en pratique privée et n'est pas lié par un contrat de travail à l'hôpital. Si ces conditions sont remplies, il peut facturer une visite, avec un supplément de 50\% sur la composante médicale, et le tout par période de 5 minutes en fonction du temps consacré; à cela peuvent s'ajouter des prestations spéciales, également avec un supplément de $50 \%$ sur la partie médicale de ces prestations.

Les pédiatres peuvent suivre actuellement une formation en ultrasonographie de la hanche et de l'abdomen (SVUPP). Cette formation sera-t-elle toujours reconnue pour l'obtention de la valeur intrinsèque qualitative nécessaire pour pouvoir facturer ces actes selon le TarMed?

Que signifie FA pédiatrie du développement? Le simple fait d'être pédiatre FMH suffira-t-il à pouvoir facturer un examen approfondi du développement en dehors des visites de routine de la SSP?

Tout pédiatre semble être à même de pratiquer un examen neurologique approfondi de l'enfant? Dès lors, pourquoi limiter le droit d'être remboursé pour un tel examen aux seuls neuropédiatres?

Je suis stupéfait du peu d'articles pédiatriques dans votre catalogue! Comment l'expliquer?

M.-A. P. $\dot{a} V$.
Il n'y a aucun lien entre le revenu et l'importance d'une discipline, d'une part, et le nombre de positions dans la structure tarifaire TarMed, d'autre part. Une position du genre "prestation pédiatrique à $1000 \mathrm{fr}$. l'heure» contenterait certainement tous les pédiatres. Mais elle n'est bien sûr pas possible. En fait:

1. L'attestation de spécialisation $(=F A=$ Fachausweis) en ultrasonographie de la hanche chez les nouveau-nés, prescrite par la loi, est également valable dans les tarifs TarMed, sans restriction.

2. L'attestation de spécialisation en pédiatrie du développement doit encore être créée; d'ici là, tous les pédiatres peuvent facturer la prestation et également les médecins bénéficiant de la garantie des droits acquis.

Pour conclure: les positions que remettez en question ou que vous critiquez ont toutes été élaborées avec les délégués de votre société de discipline médicale.

Comment sont définies les différentes salles de radiologie, d'échographie (grande/petite)?

P.W. $\dot{a} K$.

Il s'agit en fait de secteurs et non de salles. Ils comprennent pour l'essentiel les coûts d'investissement des appareils et les frais de personnel, en plus du matériel de consommation courante, etc. Notre service tarifaire pourra vous donner plus de détails à ce sujet.

\section{Kampagne "Feel Your Power»}

Sehr geehrter Herr Präsident

Sehr geehrte Frau Dr. Perrin

Die Verbindung der Schweizer Ärztinnen und Ärzte FMH hat mit einigem Erstaunen und Befremden von Ihrer Kampagne «Feel Your Power» Kenntnis genommen. Die Reaktion unserer Basis war, wie E-mails, die selbst am Sonntag eingetroffen sind, bezeugen, weit weniger verhalten: Einstimmig wurde nur Unverständnis, Kopfschütteln und nicht selten Empörung signalisiert.

In der Sache halten wir folgendes fest:

Präventionsstrategien müssen die gesamte Bevölkerung und deren mentale Struktur hinsichtlich der zu ändernden Lebensgewohnheiten erreichen. Wir zweifeln sehr, dass die nun laufende Medienkampagne diesem Ziele dienen kann. Wir sind ganz klar der Auffassung, dass ein grösserer Teil unserer Bevölkerung mit dieser ironisch-sarkastischen Vorgehensweise schlicht überfordert ist.

Dass im neuen Jahrtausend zweifelsohne nichts, aber auch gar nichts mehr heilig ist, daran haben wir uns ja alle gewöhnt. In diesem Sinne kann der gute Dr. Luzi Fehr in einer Gruppe von abgefuckten Trendsettern wohl die absolut coole und geile Nummer sein (wie Sie sehen, sind wir durchaus in der Lage, uns sprachlich an Sie ANZUPASSEN); ob der Wahrheitsfindung und dem gesundheitlichen Wohl unserer Bevölkerung aber damit gedient ist, muss in auch sehr distanzierter Betrachtung doch als fraglich bezeichnet werden.

Mit freundlichen Grüssen

FMH

Dr H. H. Brunner, Präsident F.-X. Deschenaux, Generalsekretär 


\section{Facharztprüfung zur Erlangung des Facharzttitels FMH für Neuropädiatrie}

Aufgrund des Weiterbildungsprogrammes, welches am 1. Januar 1995 in Kraft gesetzt wurde, ist die Teilnahme an der Facharztprüfung für diejenigen Kandidatinnen und Kandidaten Voraussetzung für die Erlangung des Facharzttitels FMH für Neuropädiatrie, welche ihre Weiterbildung in Neuropädiatrie bis am 31. Dezember 1995 nicht abgeschlossen hatten. Es empfiehlt sich, die Facharztprüfung frühestens im letzten Jahr der reglementarischen Weiterbildung abzulegen (Art. 22 WB0).
Ort und Datum: werden nach Eingang der Anmeldungen, abhängig von gewünschter Prüfungssprache, festgelegt.

Prüfungsgebühr: Die Fachgesellschaft erhebt eine Prüfungsgebühr von Fr. 400.-.

Anmeldung: unter Beilage des Curriculums an: Prof. E. Boltshauser, Uni-Kinderklinik, Abteilung Neurologie, Steinwiesstrasse 75, 8032 Zürich, Tel. 0126673 30, Fax 012667163.

Anmeldefrist: 31. Mai 2000

\section{Examen de spécialiste en vue de l'obtention du titre de spécialiste FMH en neuropédiatrie}

Selon le programme de formation postgraduée entré en vigueur le $1^{\text {er }}$ janvier 1995, la participation à l'examen de spécialiste est une condition requise pour les candidats à l'obtention du titre de spécialiste FMH en neuropédiatrie qui n'avaient pas terminé leur formation postgraduée en neuropédiatrie le 31 décembre 1995. Il leur est recommandé de passer l'examen de spécialiste au plus tôt durant la dernière année de leur formation postgraduée réglementaire (art. 22 RFP).
Lieu et date: Le lieu ainsi que la date de l'examen seront communiqués après réception des inscriptions et en fonction de la langue dans laquelle le candidat souhaite passer l'examen.

Taxe d'examen: La société de discipline médicale prélève une participation aux frais de Fr. 400.-.

Inscription: accompagnée de votre curriculum auprès du Prof. E. Boltshauser, Uni-Kinderklinik, Abteilung Neurologie, Steinwiesstrasse 75, 8032 Zurich, tél. 0126673 30, fax 012667163.

Délai d'inscription: le 31 mai 2000 


\section{Facharztprüfung zur Erlangung des Facharzttitels FMH für Neuroradiologie}

Aufgrund des Weiterbildungsprogrammes, welches am 1. Januar 1996 in Kraft gesetzt wurde, ist die Teilnahme an der Facharztprüfung für diejenigen Kandidatinnen und Kandidaten Voraussetzung für die Erlangung des Facharzttitels FMH für Neuroradiologie, welche ihre Weiterbildung in Neuroradiologie bis am 31. Dezember 1996 nicht abgeschlossen hatten. Es empfiehlt sich, die Facharztprüfung frühestens im letzten Jahr der reglementarischen Weiterbildung abzulegen (Art. 22 WBO).
Ort: Zürich

Datum: Mittwoch, 22. November 2000

Der genaue Ort und die Prüfungszeit werden nach Eingang der Anmeldungen bekanntgegeben.

Prüfungsgebühr: Die Fachgesellschaft erhebt eine Prüfungsgebühr von Fr. 300.-.

Anmeldung: PD Dr. W. Wichmann, Neuroradiologisches Institut, Klinik im Park, Seestrasse 220, 8027 Zürich.

Anmeldefrist: 30. Juli 2000

\section{Examen de spécialiste en vue de l'obtention du titre de spécialiste FMH en neuroradiologie}

Conformément au programme de formation postgraduée entré en vigueur le 1 janvier 1996, la participation à l'examen de spécialiste est obligatoire pour les candidats à l'obtention du titre de spécialiste FMH en neuroradiologie qui n'avaient pas encore terminé leur formation postgraduée en neuroradiologie le 31 décembre 1996. Il est recommandé de passer l'examen de spécialiste au plus tôt durant la dernière année de formation postgraduée réglementaire (art. 22 RFP).
Lieu: Zurich

Date: le mercredi 22 novembre 2000

Le lieu et l'horaire de l'examen seront communiqués après réception des inscriptions.

Taxe d'examen: La société de discipline médicale prélève une participation aux frais de Fr. 300.-.

Inscription: Dr W. Wichmann, p.-d., Neuroradiologisches Institut, Klinik im Park, Seestrasse 220, 8027 Zurich.

Délai d'inscription: le 30 juillet 2000 


\section{Facharztprüfung zur Erlangung des Facharzttitels FMH für Endokrinologie-Diabetologie}

Aufgrund der Weiterbildungsprogramme, welche am 1. Januar 1997 in Kraft gesetzt wurden, ist die Teilnahme an der Facharztprüfung für diejenigen Kandidatinnen und Kandidaten Voraussetzung für die Erlangung des Facharzttitels FMH für Endokrinologie-Diabetologie, welche ihre Weiterbildung in Endokrinologie-Diabetologie bis am 31. Dezember 1997 nicht abgeschlossen hatten. Es empfiehlt sich, die Facharztprüfung frühestens im letzten Jahr der reglementarischen Weiterbildung abzulegen (Art. 22 WBO).
Ort: Zürich

Datum: Freitag, 1. Dezember 2000 (schriftliche Prüfung am Vormittag, mündliche Prüfung am Nachmittag)

Prüfungsgebühr: Die SGED erhebt eine Prüfungsgebühr von Fr. 500.-.

Anmeldung:

Innere Medizin: Mme Christel Robert-Tissot, Division d'Endocrinologie et Diabétologie, Hôpital Universitaire de Genève, 1211 Genève 14, Tel. 022 37293 02, Fax 0223729326.

Kinder- und Jugendmedizin: Kinderspital Zürich, z.Hd. Sekretariat Endokrinologie/Diabetologie, Steinwiesstrasse 75, 8032 Zürich, Tel. 0126673 09, Fax 012667983.

Anmeldefrist: 15. September 2000

\section{Examen de spécialiste en vue de l'obtention du titre de spécialiste FMH en endocrinologie- diabétologie}

Conformément aux programmes de formation postgraduée entrés en vigueur le $1^{\text {er }}$ janvier 1997, la participation à l'examen de spécialiste est une condition requise pour les candidats à l'obtention du titre de spécialiste en endocrinologie-diabétologie qui n'avaient pas encore terminé leur formation postgraduée en endocrinologie-diabétologie le 31 décembre 1997. Il leur est recommandé de passer l'examen de spécialiste au plus tôt durant la dernière année de leur formation postgraduée réglementaire (art. 22 RFP).

\section{Lieu: Genève}

Date: Vendredi, $1^{\mathrm{er}}$ décembre 2000 (examen écrit le matin, examen oral l'après-midi)

Taxe d'examen: La SSED prélève une participation aux frais de Fr. 500.-.

\section{Inscription:}

Médecine interne: Mme Christel Robert-Tissot, Division d'Endocrinologie et Diabétologie, Hôpital Universitaire de Genève, 1211 Genève 14, Tel. 022 37293 02, Fax 0223729326.

Pédiatrie: Kinderspital Zürich, z.Hd. Sekretariat Endokrinologie/Diabetologie, Steinwiesstrasse 75, 8032 Zurich, Tel. 0126673 09, Fax 012667983.

Délai d'inscription: le 15 septembre 2000 